\title{
Cáncer de mama en Chile. Un aporte clínico y epidemiológico según un registro poblacional metropolitano: 1.485 pacientes*
}

\author{
Drs. IVÁN SERRA C. ${ }^{1}$, RAÚl MARTÍNEZ R. ${ }^{1}$, XIMENA MIMICA S. ${ }^{1}$, \\ GABRIEL CAVADA CH. ${ }^{2}$, CLAUDIA AGUAYO S. ${ }^{3}$ \\ Servicios de Cirugía y Urgencia, Hospital Sótero del Río, Santiago, Chile. \\ Estadístico, Escuela de Salud Pública, Universidad de Chile, Santiago, Chile. \\ 3 Epidemióloga, Hospital Regional de Concepción, Concepción, Chile.
}

\section{Abstract}

\section{Analysis of 1,485 women treated for breast cancer in two public hospitals}

Background: Breast cancer was included as a priority in the Chilean health reform, providing universal and guaranteed access to diagnosis and treatment to all women aged 15 years or more. Aim: To analyze the incidence, staging and survival of breast cancer in a cohort of women. Material and Methods: Retrospective analysis of medical records of women operated for breast cancer between 1994 and 2005 in two public hospitals of Metropolitan Santiago. Incidence rates were calculated using statistical information provided by the Ministry of Health. Results: The clinical records of 1.485 women aged 18 to 99 years (median 55 years), were analyzed. The number of lesions detected in early stages (T1 N0), increased progressively over time. The number of lesions in advanced stages was constant along time, but its proportion decreased progressively. The overall fifteen years survival of operated women was $64 \%$. Survival was significantly better among women without lymph node involvement. The incidence rates of breast cancer increased steadily during the study period. Mortality slightly increased during the nineties but is decreasing during the present decade, reaching a rate of 12.2 per 100.000 women during 2005. Conclussion: In this series of patients, the diagnosis of breast cancer in early stages increased in the last decade, resulting in better survival rates.

Key words: Breast cancer, survival, incidence.

\section{Resumen}

El objetivo de este estudio es entregar un aporte clínico y epidemiológico al relevante problema del cáncer de mama en Chile, en base al registro poblacional o casuística del Servicio de Salud Metropolitano Sur Oriente (SSMSO). Se analizan 1.485 mujeres atendidas entre 1994 y 2005 en el Hospital Sótero del Río y entre 2003 y 2005 en el Hospital Padre Hurtado. En los resultados se presenta la frecuencia de este cáncer en Chile y nuestro servicio, además de su comparación con otros países. En el SSMSO destaca el incremento progresivo, absoluto y relativo, de cánceres incipientes y la mantención absoluta con disminución

* Recibido el 28 de Julio de 2009 y aceptado para publicación el 4 de Septiembre de 2009.

Correspondencia: Dr. Iván Serra C.

Concha y Toro 3459. Puente Alto, Santiago, Chile.

E-mail: ivan.serra.canales@gmail.com 
relativa de cánceres avanzados. Esto es parecido en los dos hospitales pero inferior a lo observado en las pacientes semi-privadas del Sótero del Río, en las cuales predominan los incipientes. La sobrevida global a 15 años sobrepasa el 63\% y es significativamente mayor en las enfermas sin compromiso linfonodal. La mortalidad por cáncer de mama en el SSMSO se elevó moderadamente durante los 90 pero ha bajado en forma moderada en esta década; actualmente es la más baja de la Región Metropolitana y bastante inferior al promedio nacional. Se plantea la hipótesis de que la razón que explica la baja mortalidad por cáncer de mama en el SSMSO, es su alta proporción de cánceres incipientes lo cual a su vez puede deberse a una buena coordinación con la atención primaria y al aumento de mamografías y ecografías mamarias. La principal conclusión es la utilidad de tener un registro de cáncer.

Palabras clave: Cáncer de mama, epidemiología, etapificación, sobrevida, pronóstico.

\section{Introducción}

El cáncer de mama presenta una frecuencia muy diferente según los países, siendo alta en los desarrollados y baja en los no industrializados ${ }^{1}$. También la tendencia al cambio es variable. En Japón, Singapur y China ha estado aumentando desde la década de los $70^{2-4}$, mientras en Estados Unidos después de una estabilización entre el 2000 y 2003 ha comenzado a declinar ${ }^{5}$, pero con una tasa cruda 3 veces superior a la chilena. El aumento de frecuencia de los cánceres en Francia entre 1980 y 2006 se debe fundamentalmente a las localizaciones de mama y pulmón en las mujeres, además de próstata en los hombres ${ }^{6}$. En Sudamérica, Argentina y Uruguay, con un nivel de desarrollo similar al nuestro, la incidencia de cáncer de mama es el do$b^{7}{ }^{7}$. Este contraste respaldaría la existencia de factores de riesgo diferentes en estos dos países, donde, entre otros factores explicativos, se podría postular para Chile una paridad que ha sido hasta hace algunos años más alta y el primer embarazo a una edad más temprana.

La mortalidad por este cáncer es algo más de la mitad de la observada en países desarrollados ${ }^{8}$, lo cual sugiere que la proporción de cánceres incipientes en Chile es bastante más baja que en países con un mayor desarrollo de sus sistemas de salud y una población más educada, asumiendo que las normas de tratamiento y su cumplimiento son similares. Nuestra tasa de mortalidad ha aumentado moderadamente en los últimos 15 años pero, lamentablemente no es posible comparar con la incidencia, la cual se informa referida sólo a los hospitales públicos, excluyendo incluso lo referente a Fuerzas Armadas y servicios delegados pertenecientes también al sector público de salud ${ }^{8}$. Por otra parte, se trata sólo de listados y en consecuencia, no disponemos de registros de cáncer verdaderos constituyendo la información nacional entregada a los organismos internacionales apenas una estimación parcial y discutible de la realidad.

La carga de enfermedad por cáncer de mama en
Chile es alta si se mide por consultas, hospitalizaciones e intervenciones quirúrgicas, además de necesitar en la mayoría de los casos tratamientos largos y de alto costo, especialmente radioterapia y quimioterapia. Por ello, incluso la ampliación de programas de tamizaje resultaría costo-efectiva9. Debe considerarse, sin embargo, que no todas las pacientes con cáncer de mama mueren por esta causa: un número importante son de edad avanzada y fallecen por otras enfermedades (neumopatías, cardiovasculares, diabetes mellitus) además de que el cáncer de mama podría ser menos agresivo en personas añosas; también algunas fallecen a consecuencia de otros cánceres e incluso leucemia aguda en relación posible con el tratamiento ${ }^{10}$.

El objetivo de este trabajo es entregar, en base al registro poblacional o casuística del Servicio de Salud Sur Oriente de la Región Metropolitana (SSMSO), un aporte tanto epidemiológico como clínico al relevante problema de salud que constituye el cáncer de mama en Chile, como inicio de un esfuerzo para entregar información más completa y analítica en un futuro cercano.

\section{Material y Método}

Se trata de un estudio descriptivo y retrospectivo iniciado con una revisión de la literatura internacional y chilena sobre cáncer mamario, tanto en lo epidemiológico como lo clínico. Las publicaciones chilenas citadas en forma muy parcial incluyen tres tesis de magíster en salud pública desarrolladas en los últimos 20 años ${ }^{11-13}$. No se han considerado en este análisis los cánceres de mama masculinos ni se ha establecido una segregación por origen étnico. No se analiza las variables de tipo hormonal ni otros factores de riesgo conocidos como tampoco los tratamientos efectuados, los cuales han seguido las normas establecidas.

Para la incidencia y mortalidad internacional se han seleccionado países por su calidad de desarrollados en Europa Occidental, Oceanía y Norteamé- 
rica. Se eligen algunos de Sudamérica por el contraste entre ellos, consignando para Chile las cifras entregadas por el Ministerio de Salud (Minsal), aunque referidas sólo al Sistema Nacional de Servicios de Salud (SNSS) con la base poblacional limitada a las mujeres adscritas al Fondo Nacional de Salud (Fonasa) $)^{14}$.

La incidencia de cáncer mamario para Chile de los últimos años, se calcula con el agregado de una estimación publicada para el sector privado, sin incluir la información de las Fuerzas Armadas y servicios delegados por no existir publicaciones al respecto $^{15}$. La base poblacional son las mujeres según estimación del Instituto Nacional de Estadística (INE) aunque no es coincidente con las cifras obtenidas en los últimos censos, en los años correspondientes.

Para calcular la incidencia anual en nuestro SSMSO (1994-2005) se usó inicialmente la base de datos de una tesis de magíster en salud pública ${ }^{13}$, la cual ha sido corregida y actualizada en forma permanente, aunque los datos 2006-2008 no se presentan.

El grado de avance según estadío se ha establecido según el estudio anátomo-patológico, el cual habitualmente entrega un grado de avance inferior o mejor que el dado por la etapificación clínica. Todas las pacientes tuvieron verificación histológica.

La evolución de la incidencia de cáncer mamario, cifras absolutas y tasas, incluyen el Hospital Sótero del Río en todo el período, y el Hospital Padre Hurtado desde el 2003.

La sobrevida para las 1.485 pacientes se calcula con el método Kaplan Meier, global y según compromiso ganglionar, incluyendo la categoría III C no considerada en años anteriores, con seguimiento hasta mayo 2009.

La incidencia y mortalidad como tasas por 100.000 mujeres para nuestro Servicio de Salud se establece según nuestra base de datos y los de Antofagasta y provincia de Valdivia según una publicación del Ministerio de Salud ${ }^{16,17}$.

\section{Resultados}

La tasa de incidencia, de cáncer mamario en países desarrollados seleccionados, varió entre 82 y 101 por 100.000 mujeres en el año 2003 y la mortalidad en los mismos entre 18 y 24 por 100.000 mujeres, una razón entre 1: 4 y 1: 5, esta última en Estados Unidos, sugiriendo una frecuencia más alta de cánceres incipientes o un mejor tratamiento ya que presentan mayor sobrevida. En los países sudamericanos, enfatizando la falta de registros nacionales que hace que sus incidencias sean sólo estimacio- nes, las tasas comunicadas variaron entre 44 y 74 por 100.000 mujeres para el mismo año, una razón entre 1: 3 y 1: 3,5 aunque dejando aparte a Uruguay que tiene un registro nacional y se asemeja a los países desarrollados (Tabla 1).

Se puede proponer una estimación de incidencia más verdadera y completa considerando el sector privado, lo cual proporciona cifras de incidencia menores a las estimadas por una unidad del Ministerio de Salud. Nuestra propuesta entrega una incidencia menor que sería más real, del orden de 30 por 100.000 mujeres y ascenso de 27 a 32 por 100.000 mujeres entre 1999 y 2003 (Tabla 2).

La mortalidad por cáncer de mama en Chile en los últimos 15 años muestra un moderado ascenso desde 12,3 por 100.000 mujeres en 1990 a 13,8 el año 2006, significando este último dato la cantidad de 1.045 mujeres fallecidas por esta causa (Figura 1).

Tabla 1. Incidencia y mortalidad por cáncer mamario en el mundo, 2003. Tasas por 100.000 mujeres

\begin{tabular}{lcc}
\hline Ca mama & Incidencia & Mortalidad \\
\hline EE.UU. & 101,1 & 19,0 \\
Francia & 91,9 & 21,5 \\
Inglaterra & 87,2 & 24,3 \\
Canadá & 84,3 & 21,1 \\
Australia & 83,2 & 18,4 \\
Suiza & 81,7 & 19,8 \\
Uruguay & 83,0 & 24,1 \\
Argentina & $74 *$ & 21,8 \\
Brasil & $46 *$ & 14,1 \\
Chile & $44 * *$ & 13,3 \\
\hline
\end{tabular}

*Valores estimados.

**Valor estimado para sector público (SNSS).

Tabla 2. Incidencia anual de cáncer de mama en Chile. 1999-2003. Tasas por 100.000 mujeres

\begin{tabular}{cccc}
\hline Años & $\begin{array}{c}\mathbf{N}^{\mathbf{0}} \text { casos } \\
\text { SNSS }\end{array}$ & $\begin{array}{c}\mathbf{N}^{\mathbf{0}} \text { casos } \\
\text { sector } \\
\text { privado* }\end{array}$ & $\begin{array}{c}\text { Tasa } \\
\text { global de } \\
\text { incidencia }\end{array}$ \\
\hline 1999 & 1.693 & 400 & 27,3 \\
2000 & 1.538 & 400 & 24,9 \\
2001 & 1.877 & 400 & 29,0 \\
2002 & 2.013 & 400 & 30,3 \\
2003 & 2.160 & 400 & 31,8 \\
\hline
\end{tabular}

* Valores estimados para el sector privado (Ref. 15) 


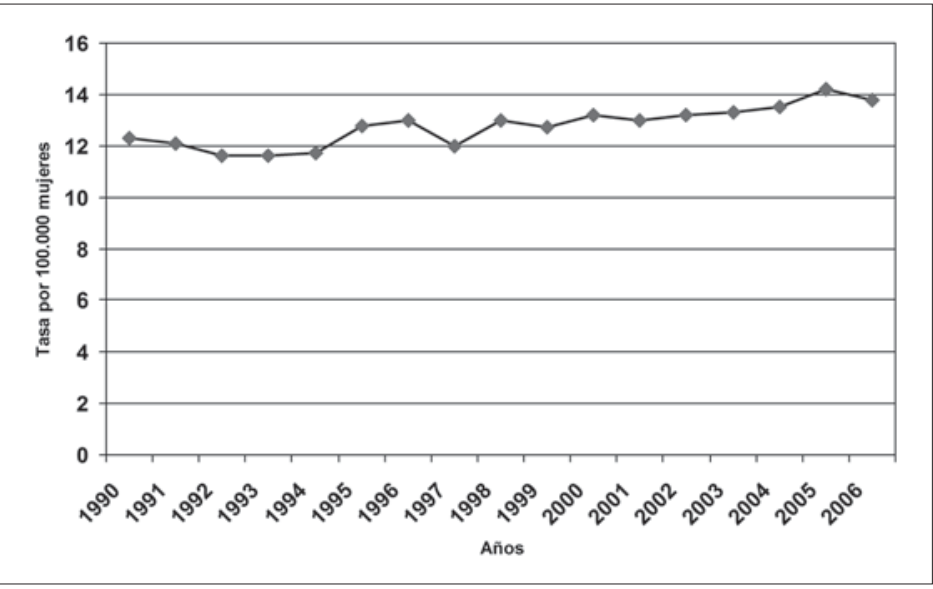

Figura 1. Mortalidad por cáncer de mama en Chile 1990- 2006. Tasas por 100.000 mujeres.
La revisión de esta casuística permitió corregir la estadificación en cuatro pacientes calificadas inicialmente como estadíos I que pasaron a II A por tener compromiso linfonodal. La incidencia absoluta de cánceres de mama en el SSMSO en el período estudiado muestra un incremento importante y mantenido desde 59 pacientes en 1994 hasta 206 pacientes el 2005. Los últimos tres años, 2006 a 2008, presentan casi 300 casos anuales.

La edad promedio de las 1.485 pacientes fue de 56,3 años, relativamente estable en el período analizado de 12 años. La mediana fue de 55 años con rango entre 18 y 99 años. Sólo el 3,5\% estuvo bajo 35 años pero un relevante $30 \%$, (428 enfermas), tenía 65 años o más (datos no mostrados).

La estadificación de las 1.485 pacientes con cán- cer de mama muestra que los cánceres in situ en los primeros años del período 1994-2005 fueron muy escasos, aumentando en la década del 2000. Los cánceres incipientes TI N0 también aumentan progresivamente en el tiempo, siete veces más que lo observado en la década del 90. Los cánceres avanzados se mantienen en forma absoluta, pero su proporción disminuye por el aumento de pacientes atendidas. Se ha considerado el grupo III C, el cual no se consignaba inicialmente. Sólo tres enfermas no han sido aún estadificadas por carencia de las biopsias definitorias correspondientes. La tasa de incidencia subió de 9,7 por 100.000 mujeres en 1994 a 28,6 el 2005, lo cual refleja una gran mejoría en la captación de pacientes por nuestros dos hospitales (Tabla 3).

Tabla 3. Incidencia anual y acumulada de cáncer de mama según estadíos. SSMSO, 1994-2005

\begin{tabular}{|c|c|c|c|c|c|c|c|c|c|c|c|c|c|}
\hline Estadíos & 1994 & 1995 & 1996 & 1997 & 1998 & 1999 & 2000 & 2001 & 2002 & 2003 & 2004 & 2005 & Total \\
\hline 0 & 0 & 0 & 1 & 2 & 3 & 3 & 7 & 6 & 8 & 11 & 18 & 12 & 71 \\
\hline I & 7 & 9 & 3 & 6 & 7 & 11 & 19 & 27 & 32 & 34 & 36 & 40 & 231 \\
\hline II A & 18 & 7 & 11 & 16 & 22 & 23 & 50 & 22 & 30 & 43 & 56 & 65 & 363 \\
\hline II B & 11 & 17 & 13 & 17 & 20 & 20 & 26 & 36 & 35 & 54 & 34 & 38 & 312 \\
\hline III A & 5 & 8 & 12 & 15 & 11 & 17 & 18 & 23 & 9 & 14 & 18 & 14 & 164 \\
\hline III B & 10 & 8 & 7 & 17 & 25 & 5 & 16 & 18 & 15 & 12 & 14 & 18 & 165 \\
\hline III C & 2 & 5 & 1 & 6 & 8 & 10 & 2 & 10 & 20 & 12 & 17 & 15 & 118 \\
\hline IV & 6 & 2 & 3 & 5 & 4 & 10 & 5 & 2 & 6 & 4 & 8 & 4 & 59 \\
\hline$X$ & 0 & 0 & 0 & 0 & 2 & 0 & 0 & 0 & 0 & 0 & 1 & 0 & 3 \\
\hline Total & 59 & 56 & 51 & 84 & 102 & 99 & 143 & 144 & 155 & 184 & 202 & 206 & 1.485 \\
\hline Incidencia & 9,7 & 9,6 & 9,6 & 14 & 16,6 & 15,9 & 21,9 & 20,3 & 23,5 & 27,3 & 29,6 & 28,6 & 18,9 \\
\hline
\end{tabular}

X: Aun no etapificados. 
El cálculo de las tasas de incidencia según comunas muestra una gran diferencia entre las dos rurales, San José de Maipo y Pirque, ambas con escasa población y alta incidencia, en comparación con las comunas urbanas que tienen hasta un $50 \%$ menos de incidencia. La diferencia de estas dos comunas con el resto es muy significativa (datos no mostrados).

La comparación de cánceres in situ operados en el Hospital Sótero del Río y Hospital Padre Hurtado, muestra una proporción similar en ambos $(4,2 \mathrm{y}$ $4,7 \%$ ) pero en la atención semi-privada del Sótero del Río se observa 8,9\%. Los casos T1 N0 también son más frecuentes en lo privado institucional: $33,9 \%$ comparado con 14,8 en lo institucional. La proporción de pacientes T1N0 fue de $22,3 \%$ en el Sótero del Río.

En el período analizado fallece un tercio de nuestras pacientes, siendo la sobrevida global de $63,6 \%$ a 15 años (Figura 2). La evolución, mortalidad y sobrevida son por supuesto muy diferentes según el estadío.

Menos de un quinto de las pacientes $(19 \%)$ no tuvo exploración axilar $(\mathrm{n}=288)$, por tratarse de cánceres in situ o en etapa IV. De 1.197 pacientes sometidas a dicha exploración, la sobrevida, en los casos sin compromiso linfonodal $(n=599)$ fue significativamente más elevada, tanto a 5 como a 10 y 15 años (Figura 3).

Las cifras absolutas y tasas de incidencia suben en forma constante en los 12 años estudiados pero la mortalidad tiende a subir moderadamente en la década del 90, con algunos altibajos, hasta una tasa de 13,3 por 100.000 mujeres en 1999 (las cifras absolutas aumentan pero la tasa menos, dado el aumento vegetativo de la población, incrementado mas en en el SSMSO por inmigración). En la década actual, la mortalidad por cáncer de mama en este servicio ha tendido a bajar hasta una tasa de 12,2 el 2005 (Fig 4). El 2006 fué incluso 10,7 , la más baja de la región metropolitana y bastante inferior a la nacional, 13,8 ese año El rango de los otros cinco servicios metropolitanos varió el 2006 entre 14,2 y 21,9.

Finalmente, la incidencia de cáncer

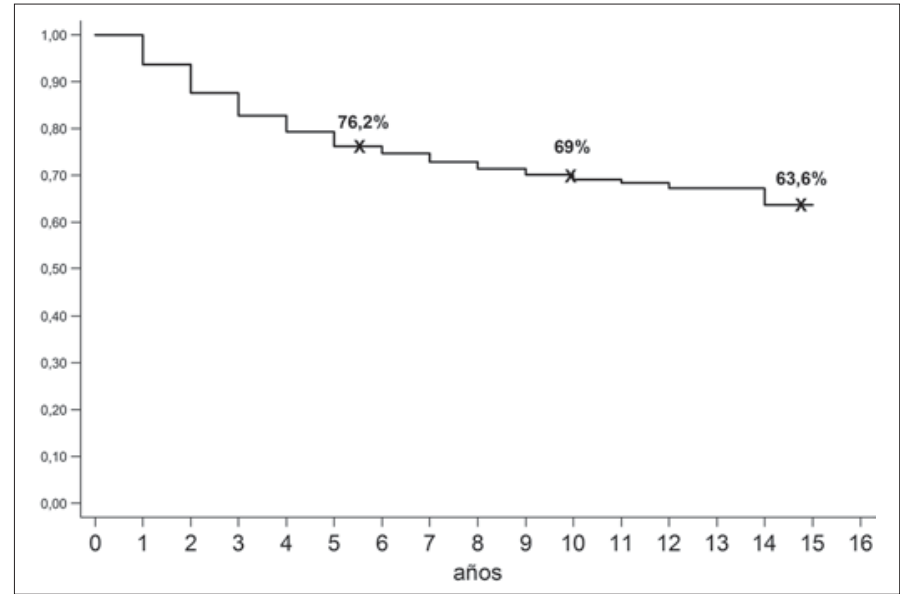

Figura 2. Sobrevida global a 5, 10 y 15 años (Kaplan Meier), SSMSO 1994-2009.

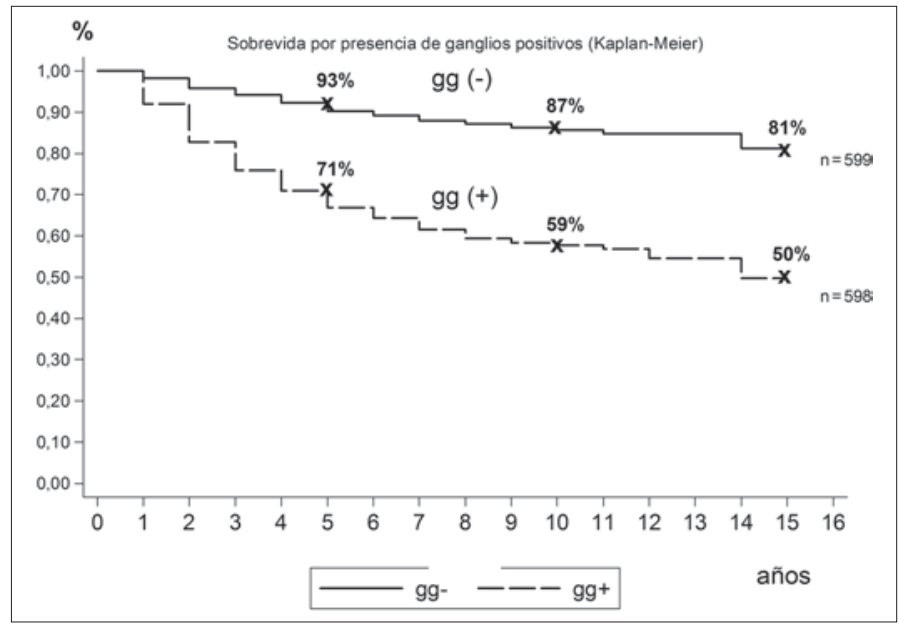

Figura 3. Sobrevida alejada según ganglios positivos o negativos, SSMSO 1994-2005.

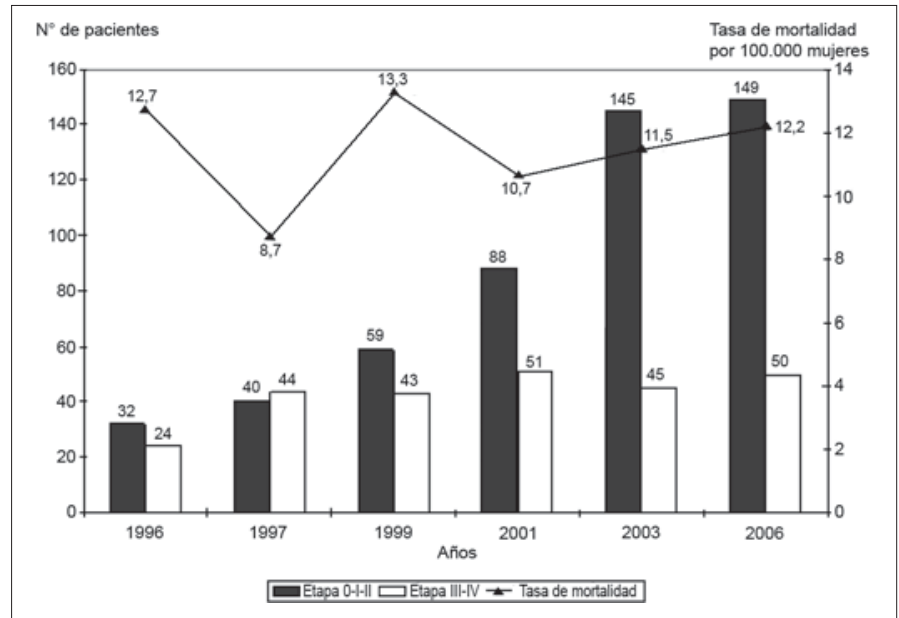

Figura 4. Incidencia y mortalidad por cáncer de mama según estadíos, SSMSO 1995-2005. 


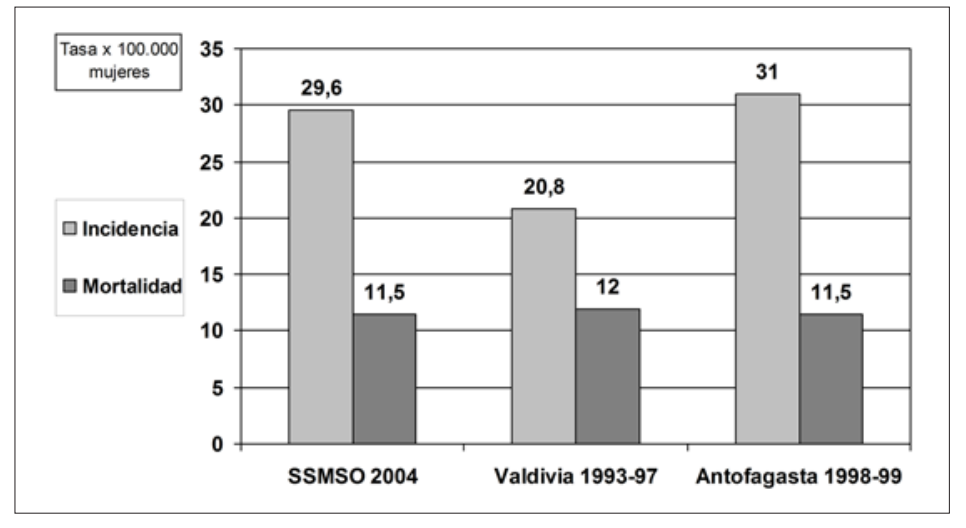

Figura 5. Incidencia y mortalidad por cáncer mamario en poblaciones de tres Servicios de Salud: Metropolitano Sur Oriente, Valdivia y Antofagasta. mamario en nuestro Servicio es similar a la de los únicos otros dos registros poblacionales del país, Antofagasta y Valdivia, al igual que la mortalidad (Figura 5).

\section{Discusión}

Este estudio tiene la limitación en lo epidemiológico referido a Chile de que cualquier incidencia que se utilice es sólo una estimación. Creemos que nuestra propuesta se acerca más a lo real al incluir el sector privado (como estimación) y usar como base poblacional la global del país. Lo informado por una unidad del Ministerio de Salud usa como numerador lo correspondiente al SNSS y como base poblacional el $67 \%$ de las mujeres, sólo las adscritas a Fonasa ${ }^{14}$, a pesar del conocimiento de que muchas personas se atienden en el sector público sin la cotización correspondiente. Esto ha sido ratificado por un estudio de Cepal del año 2000, el cual mostró que el número de usuarios que no pertenecen al sistema público ni al de seguros privados de salud (Isapres) fue creciente en la década del 90 desde 1.336 .021 ó $10 \%$ de toda la población en 1990, hasta hasta 2.717 .207 o un $18 \%$ de la población en $1999^{18}$. Esas personas no consultan en clínicas privadas sino en los hospitales públicos, como Sótero del Río y Padre Hurtado, con un certificado de indigencia, a veces temporal, dado por un consultorio de atención primaria.

La gran fortaleza del estudio es la inmensa población que cubre, sin selección alguna, en la actualidad sobre 1,5 millones de personas. Además, verificación histológica del $100 \%$ y un largo período de observación, sin perjuicio de basarse en un registro de cáncer desde el 2001. Por otra parte, existiría un número aún no cuantificado de pacientes con cáncer de mama, residentes nuestras, y, eventualmente atendidas en otros hospitales, lo cual nos hará revisar la condición de nuestra casuística como un verdadero registro poblacional.

Chile está, en lo que se refiere a cáncer de mama, muy por debajo, sin duda, de los países desarrollados con una incidencia varias veces menor y una mortalidad demasiado alta para su incidencia. Cuando hay programas de tamizaje o de pesquisa activa, la incidencia es siempre más elevada ${ }^{19}$. La razón de incidencia-mortalidad en nuestro país es baja, lo que sugiere que el problema principal es falta de captación de pacientes, especialmente cánceres incipientes.

Lo encontrado aunque no analizado en este estudio, referido a la mayor frecuencia de este cáncer en comunas rurales, es inesperado y necesita un estudio dirigido ya que la diferencia es muy significativa. El uso de agua de pozo, no clorada, en parte de la población de estas comunidades sería incluso, al revés, un factor protector ${ }^{20,21}$.

El SSMSO ha aumentado fuertemente la captación de nuevos casos pero quizás lo más importante de este estudio, es ratificar que dicho incremento se ha hecho crecientemente en base a cánceres incipientes. Esto probablemente explica que la mortalidad por cáncer de mama del SSMSO sea la más baja, además de decreciente, en la Región Metropolitana (tasa de 10,7 por 100.000 mujeres el 2006), y que la sobrevida global a 5, 10 y 15 años de esta casuística de 1.485 pacientes se compare bien con otros hospitales públicos ${ }^{12,22,23}$, no así con una clínica privada ${ }^{15}$ y un hospital público $(\mathrm{FACH})^{24}$, que atienden poblaciones seleccionadas e incluyen tamizaje.

Se ha publicado que la tasa de mortalidad por cáncer de mama en la Región Metropolitana se encuentra entre las más altas del país ${ }^{25}$, lo que es efectivo, pero en realidad existen grandes diferencias entre los Servicios de Salud que la integran: la 
del SSMSO es la mitad del que tiene la mortalidad más alta.

La comparación de nuestros datos con los disponibles de los otros dos registros poblacionales en Chile: Antofagasta y Valdivia, al mostrar incidencia y mortalidad similares entre ellos, plantea la conveniencia de un estudio comparado en detalle y en el tiempo dada la tan diferente magnitud poblacional y la carencia hasta ahora de publicaciones de tipo epidemiológico sobre estas tres poblaciones, ojalá con patrones de análisis compartidos.

Este estudio permite proponer una hipótesis para explicar la baja mortalidad por cáncer mamario en el SSMSO. Planteamos que una posible razón es la buena coordinación entre los especialistas de los dos grandes hospitales y las matronas de la atención primaria, las cuales se relacionan estrechamente con sus comunidades. Otra razón sería el aumento de mamografías y ecografías mamarias hospitalarias además de convenios municipales, junto con el desarrollo de exámenes preventivos del adulto y adulto mayor.

Lamentablemente, a pesar de que el SSMSO tiene un excelente registro de cáncer desde el 2001 no ha tenido reconocimiento del nivel central ni apoyo extranjero hasta ahora, probablemente por su posible interacción con los otros servicios de salud en una Región Metropolitana de gran población. Se sabe que hay residentes nuestras que se atienden en otros hospitales de Santiago pero lo mismo ocurre con habitantes de regiones que buscan atención en la Región Metropolitana, incluyendo nuestro servicio de salud. Sin embargo, este estudio demuestra lo importante de su desarrollo, expresado incluso en una tesis de magíster en salud pública ${ }^{13}$. En Chile se han hecho avances en estos registros ${ }^{26}$ pero sigue vigente la necesidad de resolver integralmente esta fundamental carencia sanitaria ${ }^{27}$. En lo internacional, también es un tema de preocupación pero centrada en las limitaciones y potencialidades de su uso $^{28,29}$. Un reportaje reciente publicado en una revista internacional sobre atención de pacientes oncológicos en Chile, muestra que no existe unanimidad sobre la necesidad de un registro nacional de cáncer, pero la mayoría de los especialistas lo estima imprescindible ${ }^{30}$. La experiencia del SSMSO demuestra que el costo de desarrollar registros poblacionales es moderado y su factibilidad muy alta, además de su relevante utilidad. Sin duda, la única manera de que un registro poblacional sea absolutamente válido es que sea nacional.

La verdad es que mientras no recuperemos, al menos registros regionales de cáncer confiables, se tendrá la enorme carencia de ignorar las incidencias reales. Esto presenta una gran dificultad no sólo para estudios epidemiológicos sino también para la programación y especialmente evaluación, de la atención de salud de nuestra población. Es también un problema de imagen en el aspecto internacional.

La investigación epidemiológica en cáncer de mama es relativamente escasa en nuestro medio, y la investigación clínica, aunque ofrece un número mayor de estudios, tampoco es demasiado abundante $^{31}$. Sin embargo, el problema principal del cáncer de mama en Chile, en nuestra opinión, es la carencia de un diagnóstico global e integral basado en un registro nacional que facilite la elaboración, ejecución y especialmente evaluación, de un programa para cáncer de mama factible y de alto impacto.

\section{Agradecimientos}

A las autoridades y personal de nuestro Hospital Sótero del Río y del Padre Hurtado, a la inmensa mayoría de Servicios de Anatomía Patológica de los hospitales públicos y a la totalidad de las clínicas privadas que nos facilitaron el acceso a biopsias efectuadas en esos establecimientos a pacientes residentes de nuestras comunas y atendidas en nuestro Servicio de Salud. A otros funcionarios que con dedicación y esfuerzo nos proporcionaron fichas clínicas e informaciones relevantes. Un reconocimiento especial a la Dirección del SSMSO por su apoyo permanente.

\section{Referencias}

1. Anderson BO, Caleffi M. Situación de la salud mamaria en el mundo y en Latinoamérica en particular. Rev Med Clin Condes 2006; 17: 137-141.

2. Nagata C, Kawakami N, Shimizu H. Trends in the incidence rate and risk factors for breast cancer in Japan. Breast Cancer Res Treat 1997; 44: 75-82.

3. Seow A, Duffy S, McGee M, Lee J, Lee HP. Breast cancer in Singapore: trends in incidence 1968-1992. Int J Epidemiol 1996; 25: 40-45.

4. Jin F, Shu X-O, Devesa S, Zheng W, Blot W, Gao Y-T. Incidence trends for cancers of the breast, ovary, and corpus uteri in urban Shanghai, 1972-1989. Cancer Causes Control 1993; 4: 355-360.

5. Ravdin PM, Cronin KA, Howlader N, Berg CD, Chlebowski RT, Feuer EJ, et al. The decrease in breastcancer incidence in 2003 in the United States. New Eng J Med 2007; 356: 1670-1674.

6. Guérin S, Doyon F, Hill C. The frequency of cancer in France in 2006, mortality trends since 1950, incidence trends since 1980 and analysis of the discrepancies between these trends. Bull Cancer 2009; 96: 51-57.

7. World Health Organization, World Health Statistics 
2008 (Part 2, Mortality and Burden of Disease), Ginebra. IARC. Globocan 2002.

8. Ministerio de Salud, Departamento de Estadísticas en Salud (DEIS). www/deis.minsal.cl.

9. Valencia-Mendoza A, Sánchez-González G, BautistaArredondo S, Torres-Mejía G, Bertozzi SM. Costoefectividad de políticas para el tamizaje de cáncer de mama en México. Salud Pública Mex 2009; 51 suppl 2: S 296-S 304.

10. Phillips K-A, Glendon G, Knight JA. Putting the risk of breast cancer in perspective. New Eng J Med 1999; 340: 141-144.

11. Ravera R. Epidemiología del cáncer de mama. Tesis Magíster en Salud Pública. Escuela de Salud Pública, Universidad de Chile, 1987.

12. Moreno C. Estudio de sobrevida en pacientes con cáncer de mama controladas en el Servicio de Salud Metropolitano Sur. Tesis Magíster en Salud Pública, Escuela de Salud Pública, 2001.

13. Aguayo C. Creación y explotación de un registro poblacional y hospitalario de cánceres del adulto en el Servicio de Salud Metropolitano Sur Oriente, 20012005. Tesis Magíster en Salud Pública, Escuela de Salud Pública, Universidad de Chile, 2007.

14. Prieto M, Torres S. Situación epidemiológica del cáncer de mama en Chile 1994-2003. Rev Med Clin Condes 2006; 17: 142-148.

15. Acevedo JC, Rossat C, Gutiérrez V, Reyes JM. Cáncer de mama: experiencia del Centro Integral de la Mama Clínica Las Condes. Rev Med Clin Condes 2006; 17: 248-255.

16. Goycolea M. Registro poblacional de cáncer II Región. El Vigía 2001; 14: 13-14.

17. Bertrán E, Vallebuona C, Jofré AM. Registros poblacionales del cáncer en Chile e incidencia de cáncer en la provincia de Valdivia. El Vigía 2004; 21: 26-30.

18. Rodríguez J, Tokman M. Resultados y rendimiento del gasto en el sector público de salud en Chile. CEPAL, Santiago, 2000.

19. Olsen AH, Bihrmann K, Jensen MB, Vejborg E, Hoffman J. Breast density and outcome of mammo- graphy screening: a cohort study. Br J Cancer 2009; 100: 1205-1208.

20. Wilkins JR, Comstock GW. Source of drinking water at home and site-specific cancer incidence in Washington county, Maryland. Am J Epidemiol 1981; 114: 178-190.

21. Marcus PM, Savitz DA, Millikan RC, Morgenstern H. Female breast cancer and trihalomethane levels in drinking water in North Caroline. Epidemiology 1998; 9: 156-161.

22. Peralta O, Jorquera A, Recorent C, Castillo CD, Solé J, Campodónico Y. Cáncer de mama. Resultado del programa de pesquisa y tratamiento del Servicio de Salud Central. Rev Chil Obstet Ginecol 1995; 60: 417 427

23. Pérez JA, Del Pozo M, Uribe V, Henning E, Uherek F, Salem Ch, y cols. Caracterización de 50 mujeres jóvenes con cáncer de mama. Rev Chil Cir 2000; 52: 495-502.

24. Jürgensen M, Chacón R, Baeza C, Riveros R. Cánceres de mama multifocales-multicéntricos ¿Son realmente de peor pronóstico? Rev Chil Cir 2009; 61: 125-130.

25. Vallebuona C. Registros poblacionales de cáncer. Avances en Chile. El Vigía 2001; 14: 12.

26. Roa I. La necesidad de crear registros de cáncer. Rev Chil Cir 2002; 54: 206-211.

27. Izquierdo JN, Schoenbach VJ. The potential and limitations of data from population-based state cancer registries. Am J Publ Health 2000; 90: 695-698.

28. Bickell NA, Chasing MR. Determining the quality of breast cancer care: Do tumor registries measure up? Ann Int Med 2000; 132: 705-710.

29. Peralta O. Cáncer de mama en Chile. Datos epidemiológicos. Rev Chil Obstet Ginecol 2002; 67: 439445.

30. Orellana C, Torres S, Derio L, Prieto M. Cancer care in Chile. Lancet Oncol 2003; 4: 653-656.

31. Peralta O. Diagnóstico, tratamiento y seguimiento de 1.036 mujeres con cáncer de mama en el Servicio Metropolitano Central, 2005. Información personal. Citado por Acevedo JC. Ref 15. 